\title{
SENSIBILIDADE MORAL E FATORES RELACIONADOS: PERCEPÇÃO DE ENFERMEIROS
}

\author{
Carlise Rigon Dalla Nora ${ }^{1}$, Rafaela Schaefer ${ }^{2}$, Enrique Jesús Sáez Álvarez ${ }^{3}$, María Dolores Burguete Ramos ${ }^{4}$
}

\begin{abstract}
RESUMO: Objetivou-se conhecer a percepção de enfermeiros acerca da sensibilidade moral e seus fatores relacionados. Pesquisa qualitativa, com dados coletados em grupo focal e analisado pela técnica de análise de conteúdo, em março de 2016. Participaram seis enfermeiros das seguintes áreas de atuação - cuidados paliativos, hematologia, urgência, unidade de tratamento intensivo e docente - da cidade de Valência, Espanha. Depreenderam-se quatro categorias: o conceito de sensibilidade moral; a dimensão profissional; a relação com o paciente; e a educação ética. Os enfermeiros investigados apresentaram visão restrita do conceito de sensibilidade moral, porém identificaram fatores que influenciam a sensibilidade moral. Observou-se a percepção deles em relação à necessidade de ampliação e desenvolvimento dos processos formativos em ética, ainda durante a graduação e nos próprios serviços de saúde, com vistas ao aprimoramento dos cuidados de enfermagem.

DESCRITORES: Desenvolvimento moral; Ética de enfermagem; Moral; Enfermagem.
\end{abstract}

\section{MORAL SENSITIVITY AND RELATED FACTORS: THE PERCEPTION OF NURSES}

\begin{abstract}
This study aimed to investigate nurses' perception regarding moral sensitivity and factors related to it. It is a qualitative study, with data collected in focus groups and analyzed using the technique of content analysis, in March 2016. A total of six nurses participated, from the following areas of work - palliative care, hematology, urgent care, the intensive care unit, and lecturing from the city of Valencia in Spain. Four categories were surmised: the concept of moral sensitivity; the professional dimension; the relationship with the patient; and ethical education. The nurses investigated presented a restricted view of the concept of moral sensitivity, although they identified factors which influence moral sensitivity.
\end{abstract}

DESCRIPTORS: Moral development; Ethics, nursing; Morals; Nursing.

\section{SENSIBILIDAD MORAL Y FACTORES ASOCIADOS: PERCEPCIÓN DE ENFERMEROS}

RESUMEN: Estudio cuyo propósito fue conocer la percepción de enfermeros acerca de la sensibilidad moral, así como de factores asociados a ello. Investigación cualitativa, con datos obtenidos por medio de grupo focal y analizados por la técnica de análisis de contenido, en marzo de 2016. Los participantes fueron seis enfermeros de las siguientes áreas de actuación - cuidados paliativos, hematología, urgencia, unidad de tratamiento intensivo y docente - de la ciudad de Valencia, España. Del estudio, resultaron cuatro categorías: concepto de sensibilidad moral; dimensión profesional; relación con el paciente; y educación ética. Los enfermeros investigados presentaron visión restricta del concepto de sensibilidad moral; sin embargo identificaron factores que interfieren en la sensibilidad moral. Se observó la percepción de ellos acerca de la necesidade de ampliación y desarrollo de los procesos formativos en ética aun durante la graduación y en los propios servicios de salud, a fin de que los cuidados de enfermería sean perfeccionados.. DESCRIPTORES: Desarrollo moral; Ética de enfermería; Moral; Enfermería.

${ }^{1}$ Enfermeira. Doutoranda em Enfermagem pela Universidade Católica Portuguesa. Investigadora no Instituto de Ciências da Saúde - UCP. Porto, Portugal.

${ }^{2}$ Enfermeira. Doutoranda em Enfermagem pela Universidade Católica Portuguesa. Investigadora no Instituto de Ciências da Saúde - UCP. Porto, Portugal.

${ }^{3}$ Enfermeiro. Docente da Universidade Católica de Valencia. Valência, Espanha.

${ }^{4}$ Enfermeira. Docente da Universidade Católica de Valencia. Valência, Espanha. 


\section{- INTRODUÇÃO}

A sensibilidade moral é a capacidade de conhecer determinado tema como sendo ético ${ }^{(1)}$. Os avanços da tecnologia nos cuidados de saúde têm levado ao aumento e surgimento de problemas éticos complexos e significativos no mundo. É muito importante que os enfermeiros tenham capacidade de tomar decisões éticas, a fim de defender os direitos do paciente e assegurar o respeito e a dignidade da vida $^{(2)}$.

A sensibilidade moral permite que os enfermeiros estejam conscientes dos problemas éticos da sua prática e permite a busca de soluções adequadas para a resolução desses problemas ${ }^{(3)}$. Na literatura, os termos sensibilidade moral e sensibilidade ética são encontrados muitas vezes como sinônimos. A sensibilidade ética refere-se ao conhecimento da teoria e princípios da ética, já a sensibilidade moral refere-se à atuação pessoal dentro do relacionamento interpessoal ${ }^{(4)}$. Para esse estudo, será utilizado o termo sensibilidade moral, pois se acredita que esse é um fenômeno complexo que envolve mais de uma capacidade cognitiva, envolve também as relações com o paciente e o conhecimento do contexto do problema ${ }^{(4)}$.

De acordo com a literatura, vários fatores influenciam a sensibilidade moral, sendo os mais citados: a orientação interpessoal, a estrutu ração do significado moral, a benevolência, a autonomia, o vivenciar o conflito moral e o conhecimento ${ }^{(5)}$. Além da educação ética recebida na prática e/ou na formação ${ }^{(1)}$ dos valores éticos e morais ${ }^{(6)}$ e da responsabilidade ${ }^{(4)}$. A compreensão desses fatores é fundamental para a prática ética e para o desenvolvimento de estratégias que promovam a sensibilidade moral dos enfermeiros.

Os enfermeiros se relacionam com outros sujeitos como médicos, pacientes, família, gestores entre outros, por isso precisam ter capacidade pessoal para lidar com os problemas éticos que ocorrem nessas relações interpessoais, e, portanto, necessitam ter sensibilidade moral. Nesse contexto, compreendendo que a sensibilidade moral tem uma influencia multifatorial, esse estudo teve como objetivo conhecer a percepção de enfermeiros acerca da sensibilidade moral e seus fatores relacionados.

\section{- MÉTODO}

Trata-se de estudo com abordagem qualitativa, descritiva e exploratória, com a obtenção dos dados por meio de um grupo focal. A amostra foi intencional, sendo o critério de inclusão ser enfermeiro no mínimo com cinco anos de experiência na sua área de atuação. O critério de exclusão foi ser de outra área da saúde que não enfermagem. Participaram do estudo seis enfermeiros das seguintes áreas de atuação: serviços de urgência (identificados nos depoimentos como urgência 1 e urgência 2) cuidados paliativos, hematologia, unidade de tratamento intensivo (UTI) e docente. Com o intuito de garantir a privacidade dos participantes eles foram identificados nos depoimentos de acordo com sua área de atuação.

O grupo focal foi moderado por um pesquisador experiente na realização deste tipo de técnica qualitativa. Esse moderador foi apoiado por três assistentes que atuaram como relatores e observadores. A reunião com o grupo focal foi realizada no dia 9 de março de 2016, na Universidade Católica de Valência San Vicente Mártir, na cidade de Valência, Espanha. O estudo foi realizado durante uma modalidade acadêmica do doutorado em enfermagem. Para conduzir o grupo focal foi utilizado um roteiro com perguntas abertas, desenvolvidas a partir da literatura existente sobre o tema.

No início da reunião do grupo focal, os participantes completaram um formulário com as informações relativas a características pessoais e ocupacionais, bem como a aprovação para participar do grupo focal, por meio de assinatura da declaração de consentimento livre e esclarecido. Em seguida, o moderador realizou uma breve introdução em que agradeceu a colaboração dos participantes e explicou o objetivo específico do grupo focal, sublinhando a importância de que todos expressassem livremente as suas opiniões. Reforçou-se a garantia do anonimato e a confidencialidade dos dados, bem como o direito de se retirar a qualquer momento da pesquisa. O estudo foi autorizado em três de março de 2016 pelo parecer da Universidade Católica de Valência, UCV/2015-2016/33 na Espanha e 
seguiu todos os preceitos éticos.

A reunião do grupo focal foi gravada em áudio e vídeo e, posteriormente, transcrita as gravações. Foi realizada a leitura e a releitura exaustivas das transcrições. A duração total do trabalho desenvolvido pelo grupo focal foi de uma hora e trinta minutos. Para interpretação dos dados, utilizou-se a proposta da análise de conteúdo ${ }^{(7)}$. As transcrições foram lidas e relidas, procurando ordenar o conteúdo, reestruturar as ideias e pontos-chave do material. Após a análise foi estabelecida quatro categorias que indicam a compreensão dos enfermeiros sobre a sensibilidade moral. Essas categorias foram discutidas entre os pesquisadores a fim de certificar que os resultados mostram o conteúdo manifesto nos dados.

\section{RESULTADOS}

Após a análise dos dados, quatro categorias principais foram encontradas para indicar a compreensão dos enfermeiros sobre a sensibilidade moral e seus fatores relacionados. As categorias estabelecidas foram: o conceito de sensibilidade moral; a dimensão profissional; a relação com o paciente e a educação ética.

No que se refere à caracterização dos enfermeiros, dentre os seis participantes, somente um era do sexo masculino e a faixa etária variou entre 43 e 63 anos. O tempo de formação variou de 22 a 42 anos. O tempo de trabalho como enfermeiro variou de 15 a 34 anos. Quanto ao nível de formação profissional três participantes possuíam mestrado, um era especialista e dois possuíam apenas a graduação em enfermagem.

\section{Categoria 1 - Conceito de Sensibilidade Moral}

Os resultados demonstram que os participantes falam sobre o conceito de sensibilidade moral de modo semelhante, como algo pessoal que cada um possui.

A sensibilidade moral é algo que pertence a cada um de nós, que somente somos capazes de sentir na medida somos sensíveis, a sensibilidade ou você tem ou não tem, você tem que trabalha-la. (Docente)

A sensibilidade moral seria uma coisa pessoal minha, que faz parte do meu próprio ser pessoal e que, no meu dia a dia eu vivo e que me modela mais ou menos de uma ou outra maneira em como eu posso prosseguir. (Cuidados Paliativos)

Sensibilidade é como (Paliativos) diz, é como você se vê. (Urgência 2)

Os participantes também referem que a sensibilidade moral esta ligada aos valores e as crenças pessoais do indivíduo.

A sensibilidade moral vem mais ou menos a ser os meus valores, minhas crenças, minha capacidade de decisão, ou seja, como eu tenho que viver a minha vida profissional e o que me afeta. (Cuidados Paliativos)

Falando sobre a sensibilidade e o sofrimento moral um enfermeiro comentou:

Sensibilidade é o que te causa sofrimento, se você não tiver não há sofrimento moral; então estou de acordo com o que você diz (Paliativos). Eu tenho uma especial sensibilidade moral, porque eu me trabalhei, porque eu conheço a minha personalidade. E a minha sensibilidade será mais ou menos, dependendo do que eu quiser, isso me causará mais ou menos sofrimento moral. (UTI)

Relatou-se, também, que todos os enfermeiros têm sensibilidade moral, porém alguns desenvolvem e outros não, como se evidencia nesse comentário:

Estou convencido(a) de que todos nós temos coração e que todos nós temos sensibilidade, o que acontece é que é algo que se desenvolve, é como os músculos que não se desenvolvem se não os usamos. (UTI) 


\section{Categoria 2 - Dimensão Profissional}

Essa categoria apresenta as situações relatadas pelos enfermeiros que ocorrem no âmbito da profissão, ou seja, que se relacionam com a responsabilidade profissional.

O grupo de participantes destacou gostar do seu trabalho, como se evidencia nas falas a seguir.

Quando o paciente está próximo da morte é quando eu mais gosto de trabalhar. Demorei quase a metade de uma vida profissional para saber que meu lugar é onde estou agora. (Cuidados Paliativos)

Eu não evito o trabalho porque eu gosto dele, porque eu gosto muito. (Hematologia)

Os participantes mencionam que os enfermeiros muitas vezes tomam decisões clínicas levando em consideração seus princípios e valores pessoais. Também descrevem que esses valores podem levar a conflitos com a equipe, gestão e familiares.

Os valores às vezes são diferentes dos outros profissionais com quem trabalhamos, às vezes diariamente. Então se você tem mais valores é mais fácil que você sofra. (Docente)

O que está claro é que quando você tem valores morais esses valores podem te meter em problemas burocráticos, ou com o paciente, ou até mesmo com a família. (Docente)

A responsabilidade dos enfermeiros pelos seus atos também foi um resultado evidente nas falas dos participantes.

Eu acho que existem enfermeiros que desconhecem que somos responsáveis portodos os medicamentos que aplicamos, apesar de o médico ter assinado. (Urgência 2)

Eu sempre digo aos jovens, quando em dúvida, consultar serviços de farmácia, os médicos, os supervisores e se você ainda não está seguro, continue perguntando a questão é não ficar em dúvida. (Hematologia)

Ainda sobre a responsabilidade profissional, os participantes relatam que é necessário ter sensibilidade para perceber o que está certo e o que está errado, e, por isso, referem recusar-se a prestar o cuidado quando sentem que não estão fazendo o que julgam ser correto.

Eu tenho a capacidade de discordar e discordar abertamente, quando eu vi que as coisas não estavam onde deveriam estar eu me recusei a aplicar o cuidado ou a utilização de uma droga específica ou determinada técnica, porque vai contra os meus princípios e ética profissional. (Cuidados Paliativos)

Somos capazes de nos recusar a colocar qualquer tratamento que parece não é o certo. (Urgência 1)

E a segurança quem Ihe dá? A experiência, portanto, estamos falando em que há situações em que você pode intuir que estão erradas, porque você as percebe de alguma forma, porém não tem toda a preparação para ser capaz de enfrentar outro profissional e dizer: Olha, eu isto eu não farei por isso, por isso e por isso. (Docente)

\section{Categoria 3 - Relação com o Paciente}

Essa categoria esta relacionada com o cuidado ao paciente, e os resultados expressam o respeito dos enfermeiros com a dignidade do paciente.

Ter sensibilidade moral para falar com os pacientes é imprescindível, eu penso que já a temos. (Hematologia)

Independentemente da situação extrema em que o paciente esteja ... é o doente, que merece todo o meu respeito e consideração ou seja, é a ele que devemos prestar contas. (Cuidados Paliativos)

A família também é referida pelos participantes como geradora de conflito, pois algumas vezes os valores dos profissionais e da família não coincidem.

Quando a família de alguma forma não está em harmonia com os seus próprios valores sobre o que 
fazer com o paciente. Também a enfermagem se vê com este dilema. Acontece que estamos envolvidos porque nós somos o centro de tudo. (Docente)

Os enfermeiros também demonstram respeito com o paciente, foco central do seu cuidado, referindo à necessidade de manter sempre a privacidade do paciente.

Quando chegamos na emergência não nos damos conta que o paciente é uma pessoa, isso é uma obsessão que eu sempre tive, não importa, você não precisa ver a "bunda" de todos, pode fechar as portas, fechar as cortinas, é uma obsessão que eu tenho, acho um desrespeito, a mim essas coisas me incomodam muito. (Urgência 2)

\section{Categoria 4 - Educação Ética}

Durante as discussões os participantes enfatizaram a preocupação em como preparar os estudantes - futuros profissionais de enfermagem - para desempenhar uma prática profissional com sensibilidade moral, baseada em valores e princípios éticos.

Eu preparo os jovens universitários que querem ser enfermeiros. Vocês querem falar sobre os valores que eles têm? Eles não têm nenhum [UCI], Há algo que deve haver, alguma coisa, muito pouco, muito pouco. Estamos em uma sociedade onde precisamos Ihes educar sobre estes valores. (Docente)

Os estudantes que estamos preparando agora têm menos valores... isso me assusta, porque não é que eles vão cuidar bem ou mal, é que eles não vão se dar conta, nem se quer, se eles fazem bem ou mal, porque a sua sensibilidade não existe. (Docente)

Pergunto como suprimos a falta de sensibilidade, como, como? Como nós suprimos essa ausência de valores, que não podemos generalizar, há pessoas que são muito educadas nos valores e que os valores são ainda maiores dos que os que estão aparecendo nessa discussão, mas a grande maioria não tem valores. (Docente)

\section{- DISCUSSÃO}

A partir dos resultados é possível identificar que os enfermeiros encontram situações repletas de problemas éticos em seu contexto de trabalho. Problemas éticos são questões concretas e definidas da prática de cuidados, percebidas como causa de conflito no âmbito dos valores e deveres éticos, que permitem vários cursos de ação para sua solução. Para enfrentar esses conflitos os enfermeiros necessitam reconhecer a situação como problemática, desenvolver as habilidades de sensibilidade moral e interpretação da situação, além de possuir raciocínio ético e capacidade para implementar uma ação(8).

Nesse contexto, os resultados desse estudo indicam que os participantes têm uma visão restrita sobre o conceito de sensibilidade moral, pois a referem como algo individual que cada pessoa possui, como sendo os valores e as crenças e como algo que alguns enfermeiros desenvolvem e outros não. Em nenhum momento os enfermeiros referem à sensibilidade moral como algo ligado aos aspectos relacionais, ou seja, não mencionam a influência da sua ação como enfermeiro sobre o bem-estar do paciente.

O conceito de sensibilidade moral mais utilizado na literatura atualmente é definido como "a compreensão contextual e intuitiva da situação de vulnerabilidade do paciente, tendo uma visão sobre as consequências éticas das decisões tomadas em nome deste" (9:474). Em 2006, esse conceito foi ampliado envolvendo mais dimensões do que a capacidade cognitiva, passou a incluir sensações, sentimentos, conhecimento moral e habilidades ${ }^{(4)}$.

Um estudo ${ }^{(4)}$ valida os achados desta pesquisa ao descrever que a sensibilidade não é só uma questão de feeling, ou seja, confiar nas suas próprias emoções para identificar um conflito moral, mas sim, é um componente pessoal, adquirido pela experiência de cada um, o que permite perceber o significado moral em uma determinada situação. Autores ${ }^{(9)}$ referem que a sensibilidade moral pode ser compreendida como um tipo de resposta emocional, sendo um componente pessoal necessário 
quando se lida com problemas éticos.

Os resultados desse estudo corroboram com a literatura, ao descrever que a sensibilidade moral de um indivíduo passa por um processo de desenvolvimento e mudança de forma contínua, caminhando conforme a produção da sua vida ${ }^{(10)}$. Nesse sentido, é possível dizer que todos têm sensibilidade moral, na medida em que esta é entendida como uma capacidade pessoal para lidar com o conflito ético, em situações particulares, nas relações interpessoais entre pacientes e enfermeiros ${ }^{(9)}$. A sensibilidade moral é um conceito amplo e vai muito além do que simplesmente seguir as regras e normas de uma instituição, ela permite descrever as pessoas que têm um senso inato do que é certo e do que é errado e que assumem um conjunto de atitudes éticas nas suas relações cotidianas ${ }^{(11)}$.

No que refere-se a dimensão profissional, os participantes relataram gostar do seu trabalho. Um estudo $^{(12)}$ indica que o amor e a paixão são os responsáveis pelo gostar do trabalho, associando as lembranças do seu serviço a sentimentos positivos. É importante que o trabalho possa permitir sentimentos positivos, favorecendo a realização e o crescimento pessoal dos enfermeiros.

O enfermeiro trabalha entre e com vários sujeitos envolvidos no cuidado, o que permite encontrar tensões e conflitos de valores. Estudo ${ }^{(13)}$ corrobora com os resultados ao referir que pode haver conflitos entre os valores dos enfermeiros e o conjunto de normas e valores da instituição onde trabalha, ou com familiares que tenham valores diferentes. Essas situações causam problemas éticos para os enfermeiros, podendo fazer com que estes comprometam sua integridade moral.

Os enfermeiros quando necessário recusam-se a prestar atendimento, quando entendem que não o devem fazer, bem como, assumem suas responsabilidades profissionais. Para tanto, o enfermeiro precisa ter competência profissional para mobilizar e articular saberes e valores, integrando conhecimentos no processo cotidiano ${ }^{(14)}$. Ter sensibilidade inclui a capacidade de tomar decisões éticas com inteligência e compaixão, frente a uma situação de incerteza no cuidado.

Estudo $^{(11)}$ aponta que ter enfermeiros com sensibilidade moral elevada é importante em contextos onde pode haver pressão para fechar os olhos ou ignorar as más práticas de cuidado, ou seja, com a normalização dos maus comportamentos os profissionais tornam-se acostumados e já não se opõe as más práticas nos serviços. Enfermeiros com baixa sensibilidade moral afetam negativamente o ambiente de trabalho, quando o comportamento antiético pode tornar-se normalizado ${ }^{(11)}$.

No que se refere à dimensão da relação com o paciente, a sensibilidade moral exige que o enfermeiro seja capaz de interpretar e ter consciência dos comportamentos e indícios que o paciente expressa de forma verbal ou não-verbal, a fim de determinar e compreender as necessidades do paciente ${ }^{(15)}$. Essas necessidades podem ser físicas, emocionais e espirituais e isso precisa ser levado em consideração quando se quer prestar cuidado de excelência em saúde.

De acordo com estudo(1), a sensibilidade moral requer relação com pelo menos uma outra pessoa, sendo que para existir uma relação ética eficiente entre enfermeiros e pacientes é necessário que ambos tenham uma compreensão mútua. É essa compreensão mútua que vai garantir que as decisões tomadas pelos enfermeiros sejam apreciadas pelos familiares de forma positiva.

Os participantes ressaltaram a necessidade de respeito a privacidade do paciente. Estudo ${ }^{(16)}$ de revisão valida esses resultados ao descrever que as informações sigilosas, as questões de privacidade e confidencialidade são um dos problemas éticos mais frequentes vivenciados pelos enfermeiros nos serviços de saúde. No entanto, um dos papéis mais importantes do enfermeiro é a defesa do paciente $^{(17)}$.

A importância da educação ética durante a graduação foi outro achado deste estudo. Sendo a questão central como desenvolver a sensibilidade moral nos estudantes de enfermagem. Estudos recentes indicam que a educação ética é importante para desenvolver a sensibilidade moral dos estudantes $^{(6,10,18)}$. Isso porque a sensibilidade moral é uma premissa fundamental para compreender os problemas éticos que ocorrem nos serviços de saúde e conduzir de maneira apropriada a resolução desses problemas de forma ética.

Estudo $^{(10)}$ sugere que o conhecimento ético prepara o terreno para a sensibilidade moral em um contexto profissional, já a sensibilidade moral permite que esse conhecimento seja implementado na 
prática. Ou seja, se um estudante de enfermagem tem sensibilidade moral elevada ele pode distinguir melhor os problemas éticos que ocorrem no seu serviço, tendo efeito positivo sobre o ambiente de trabalho, que será mais sensível em termos de ética.

De acordo com estudo ${ }^{(18)}$ a educação ética aumenta a qualidade do atendimento ao paciente e contribui para a profissionalização da enfermagem. A educação ética precisa promover valores, crenças e orientação ética, a fim de promover a capacidade de tomada de decisões éticas autônomas ${ }^{(19)}$.

A literatura refere que ter sensibilidade moral é uma característica importante para os estudantes de enfermagem, pois eles são os futuros profissionais que irão enfrentar situações éticas em diferentes contextos do trabalho ${ }^{(6)}$. Os resultados desse estudo refletem essa preocupação ao reconhecer a educação ética como parte essencial da educação e prática da enfermagem.

A sensibilidade moral pode ser promovida através da educação ética que consiste em métodos educacionais, como: aulas formais, conferências, seminários, análise de casos, discussão em grupos, dramatizações entre outros ${ }^{(19-20)}$. O ensino precisa considerar formas de ajudar/apoiar a transição do papel de estudante para o papel de enfermeiro, enfatizando como lidar como problemas éticos que podem resultar em sofrimento moral ${ }^{(20)}$.

Este estudo apresenta algumas limitações quanto ao pequeno número de enfermeiros participantes em um único momento de coleta de dados. Por isso, é prematuro fazer qualquer tipo de generalização dos dados sobre os fatores relacionados a sensibilidade moral dos enfermeiros, esse tema precisa ser melhor avaliado em pesquisas futuras.

\section{CONCLUSÃO}

Nesse estudo, ficou claro que os enfermeiros participantes desconhecem o conceito de sensibilidade moral adotado na literatura atual, mas por outro lado relatam categorias que estão relacionadas e que influenciam de alguma forma a sensibilidade moral dos enfermeiros, como elementos essenciais que envolvem a dimensão profissional, a relação com o paciente e a educação ética. Esse estudo sugere um quadro para futuras pesquisas que visem a explorar como podemos promover a sensibilidade moral nos enfermeiros em diferentes contextos de práticas.

Esse estudo é útil para demonstrar que discussões sobre a sensibilidade moral são fundamentais, assim como, a realização de novas pesquisas nessa área. Além disso, ressalta-se a importância da educação e sensibilização ética dos estudantes ainda durante a graduação, para que tenham uma visão ampliada do conceito de sensibilidade moral. A implicação desse estudo para a prática é significativa na medida em que a sensibilidade moral é parte integrante do processo de tomada de decisão ética nos serviços e para a prestação de atendimento de excelência.

\section{FINANCIAMENTO}

Bolsa de estudos da Coordenação de Aperfeiçoamento de Pessoal de Nível Superior (CAPES), concedida pelo Programa Ciências sem Fronteiras na modalidade de doutorado pleno no exterior, processo Bex: 2762-13-7.

\section{- REFERÊNCIAS}

1. Borhani F, Abbaszadeh A, Mohsenpour M. Nursing students' understanding of factors influencing ethical sensitivity: A qualitative study. Iran J Nurs Midwifery Res. 2013;18(4):310-5.

2. Choe K, Park S, Yoo SY. Effects of constructivist teaching methods on bioethics education for nursing students: a quasi-experimental study. Nurse Educ Today. 2014;34(5):848-53.

3. Kim YS, Kang SW, Ahn JA. Moral sensitivity relating to the application of the code of ethics. Nurs Ethics. 2013;20(4):470-8. 
4. Lützén K, Dahlqvist V, Eriksson S, Norberg A. Developing the concept of moral sensitivity in health care practice. Nurs Ethics. 2006;13(2):187-96.

5. Lützén K, Johansson A, Nordström G. Moral sensitivity: some differences between nurses and physicians. Nurs Ethics. 2000;7(6):520-30.

6. Ahn $\mathrm{SH}$, Yeom HA. Moral sensitivity and critical thinking disposition of nursing students in Korea. Int J Nurs Pract. 2014;20(5):482-9.

7. Bardin L. Análise de conteúdo. Lisboa: Edições 70; 2008

8. Robichaux C. Developing ethical skills: from sensitivity to action. Crit Care Nurse. 2012;32(2):65-72.

9. Lützén K, Evertzon M, Nordin C. Moral sensitivity in psychiatric practice. Nurs Ethics. 1997;4(6):472-82.

10. Baykara ZG, Demir SG, Yaman S. The effect of ethics training on students recognizing ethical violations and developing moral sensitivity. Nurs Ethics. 2015;22(6):661-75.

11. Jackson D, Cleary M, Mannix J. Editorial: ethical sensitivity: shaping the everyday work environment. Contemp Nurse. 2013;44(1):2-4.

12. Duarte JMG, Simões ALA. Significados do trabalho para profissionais de enfermagem de um hospital de ensino. Rev Enferm UERJ. 2015;23(3):388-94.

13. Jaafarpour M, Khani A. Evaluation of the Nurses' Job Satisfaction, and Its Association with Their Moral Sensitivities and Well-being. J Clin Diagn Res. 2012;6(10):1761-4.

14. Schaefer R, Junges JR. A construção da competência ética na percepção de enfermeiros da Atenção Primária. Rev. esc. enferm. USP. 2014;48(2):329-34.

15. Ertug N, Aktas D, Faydali S, Yalçin O. Ethical sensitivity and related factors of nurses working in the hospital settings. Acta bioeth. 2014;20(2):265-70.

16. Dalla Nora CRD, Zoboli ELCP, Vieira M. Problemas éticos vivenciados por enfermeiros na Atenção primária à saúde: revisão integrativa da literatura. Rev. Gaúcha Enferm. 2015;36(1):112-21.

17. Nogario ACD, Barlem ELD, Tomaschewski-Barlem JG, Lunardi VL, Ramos AM, de Oliveira ACC. Ações dos enfermeiros no exercício da advocacia do paciente internado em um centro de queimados. Rev. esc. enferm. USP. 2015;49(4):580-8.

18. Sahin SY, lyigun E, Acikel C. Validity and reliability of a turkish version of the modified moral sensitivity questionnaire for student nurses. Ethics \& Behav. 2015;25(4):351-9.

19. Trobec I, Starcic A. Developing nursing ethical competences online versus in the traditional classroom. Nurs Ethics. 2015;22(3):352-66.

20. Comrie RW. An analysis of undergraduate and graduate student nurses' moral sensitivity. Nurs Ethics. 2012;19(1):116-27. 